\title{
Application of Artificial Intelligence Detection System Based on Multi-sensor Data Fusion
}

\author{
https://doi.org/10.3991/ijoe.v14i06.8696 \\ Meifang Han \\ Weifang University of Science and Technology, Shandong, China \\ hmfmeifang09@21cn.com
}

\begin{abstract}
Aiming at solving the navigation and obstacle avoidance of the unmanned vehicle, the multi sensor data fusion technology and unmanned vehicle obstacle avoidance navigation algorithm were studied profoundly. According to the requirements of the application of unmanned vehicle navigation and obstacle avoidance system, multi sensor data fusion technology was applied to unmanned vehicle navigation and obstacle avoidance control system. In addition, A*VFF navigation and obstacle avoidance algorithm based on fuzzy neural network was improved. Finally, through the construction of the simulation platform, simulation experiment of the unmanned vehicle obstacle avoidance navigation was completed, and a better route was planned for unmanned vehicle in a more complex environment. The results showed that it realized the autonomous navigation of unmanned vehicle and obstacle avoidance function. Based on the above findings, it is concluded that the application of artificial intelligence detection system has good performance.
\end{abstract}

Keywords-Multi sensor data fusion; artificial intelligence; obstacle avoidance navigation algorithm

\section{$1 \quad$ Introduction}

With the development of modern science and technology, the development of computer technology, electronic technology, control technology and artificial intelligence (AI) technology, as well as automobile industry has also undergone rapid changes. To reduce traffic accidents, improve transportation efficiency, and reduce the workload of drivers, the intelligent driving technology of unmanned vehicles came into being.

Reviewing the history of automobile development, in 1970s, the total number of vehicles in the world has reached about two hundred and fifty million. Today, the total number of vehicles in the world has already exceeded one billion, and seven people have a car in average. With the rapid popularity of the vehicles, on the one hand, it has changed the living habits of human beings and provided a comfortable and convenient means of transportation for people. However, on the other hand, more and more problems have emerged, such as vehicle collision, traffic safety, traffic congestion and environmental pollution. To solve the above problems, electric vehi- 
cles, solar vehicles, artificial intelligent unmanned vehicle and other new concept vehicles came into being. Among them, the unmanned vehicle, also known as intelligent vehicle, can reduce traffic accidents and improve transportation efficiency while reducing driver's labor load, which is favored by scientists from all over the world.

The unmanned vehicle system is an intelligent agent with many functions, which has the function of environment perception, intelligent control function, and planning decision making function. The unmanned vehicle system can perform various types of driving tasks, such as road environment identification, vehicle driving state detection, vehicle driving state prediction, vehicle tracking driving, cruise control, lateral driving control, longitudinal driving control, overtaking control and so on. These auxiliary driving systems can significantly improve the safety performance of the vehicle, and make up for the fault caused by the driver's lack of technology or the error of judgment. The safety auxiliary driving system of the unmanned vehicle is different from the traditional vehicle safety protection system, such as anti-collision steel beam and airbag. It can only provide corresponding protection after the accident and it has an active safety prompting function. For vehicle driving safety, the unmanned vehicle's safety auxiliary driving system can warn the driver in advance when traffic accidents happen, avoid accidents in time, and ensure the safe driving of vehicles in the effective range. To effectively carry out vehicle identification, obstacle avoidance and tracking, it is necessary to have a real-time and reliable detection algorithm in the system.

In this paper, the multi sensor data fusion technology and research on obstacle avoidance and navigation algorithm are integrated into unmanned vehicle obstacle avoidance and navigation technology to conduct an in-depth research on unmanned vehicle. Using the camera, infrared sensors and ultrasonic sensors and observation information fused from several sensors, data integration and analysis is conducted, decision-making and evaluation are completed, and ultimately the purpose of obstacle avoidance and navigation is achieved.

\section{Literature review}

The research on intelligent control of unmanned vehicles in the United States, Germany, Germany, Italian, France and Japan has been in the world leading level. The first automated guided vehicle system was developed by the American Barret Electronics company in 1960s. YOH-HANPAO and others as stated in [1] mainly discussed the neural-net computing and the intelligent control of systems. And then it triggered the widespread concern of the western countries on the unmanned intelligent vehicles, such as the ARGO system in Italy, the NavLab system in Carnegie Mellon University (CMU), the PATH project in the US, the Demo III in the US military, the VaMoRs-P system in the German Federal National Defense University and a series of intelligent driving vehicles. Navlab series of intelligent vehicles was developed by the United States Carnegie Mellon University cooperating with other companies. Martín, and others as stated in [2] studied an intelligent vehicle based on computational perception. The system installed Sony DXC2151A camera, radar, binocular stereo vision 
sensor, GPS global positioning system, fiber optic gyro, damping differential GPS system, optical encoder and embedded computer and auxiliary equipment. In addition, Zhang and Liu as stated in [3] studied the multi-sensor data fusion technology. Through the use of multi sensor data fusion technology, this type of unmanned vehicle can adopt vision sensor and laser radar to detect pedestrians, vehicles and so on moving targets traveling on the road, and various stationary targets. As a result, autonomous navigation and obstacle avoidance and independent investigation in wild environment can be conducted.

Kim and others as stated in [4] proposed the use of radar image for autonomous driving of unmanned ground vehicles. The research on autonomous driving of the unmanned vehicle started late and developed slowly in China. The main reason is that the current research projects on the unmanned vehicle are only carried out in some universities and research institutes, but have not yet been developed to enterprises. Supported by the National 863 plan and the state's national defense research, the THMR intelligent vehicle control system has been developed by Tsinghua University computer intelligence technology and system state key laboratory. At present, THMR series has developed to THMR-V and THMR-V is made up of magnetic compass optical code locator, ultrasonic wave, GPS positioning system, laser rangefinder, twodimensional color camera and so on sensor devices. In 2017, Wang as stated in [5] proposed the design and implementation of intelligent traffic monitoring system based on Thmr - V platform. Now, autonomous unmanned vehicle detection and navigation in China mainly focus on structured roads with simple environment (like expressways), while the studies in the environment of non-structured road (like urban roads and non-grade highways) are few. Liu and others as stated in [6] studied the lane markings using orientation and vanishing point constraints in structured road scenes. Veum and others as stated in [7] discussed the sensor data fusion for soil health assessment. The result shows that the sensor data fusion is suitable for the road detection and exploration. Lin and Sun as stated in [8] applied the distributed fusion estimator for multi-sensor asynchronous sampling systems with missing measurements. Li and others as stated in [9] summarized the localization and tracking of unmanned mining equipment based on multi-sensor information fusion technology. Frikha and Moalla as stated in [10] discussed the multi-sensor data fusion based on belief function theory.

To sum up, the existing research in the field of road condition detection still needs to break through. For example, the technical reference of artificial intelligence is not very perfect. In view of the problem of artificial intelligence detection, the sensor fusion technology is proposed. In this paper, the multi sensor data fusion technology and research on obstacle avoidance and navigation algorithm are integrated into unmanned vehicle obstacle avoidance and navigation technology to conduct an in-depth research on unmanned vehicle. Therefore, this method makes up for the shortcomings of the current research on road detection and has the advantages of fast speed and accurate data. 


\section{Theoretical research}

\subsection{Multi sensor data fusion technology}

A sensor is a transmitter that corresponds to a specific purpose and can be applied to a specific occasion to transform the detected physical quantity into a recognized electrical signal. A sensor model can judge decision making is shown in Figure 1.

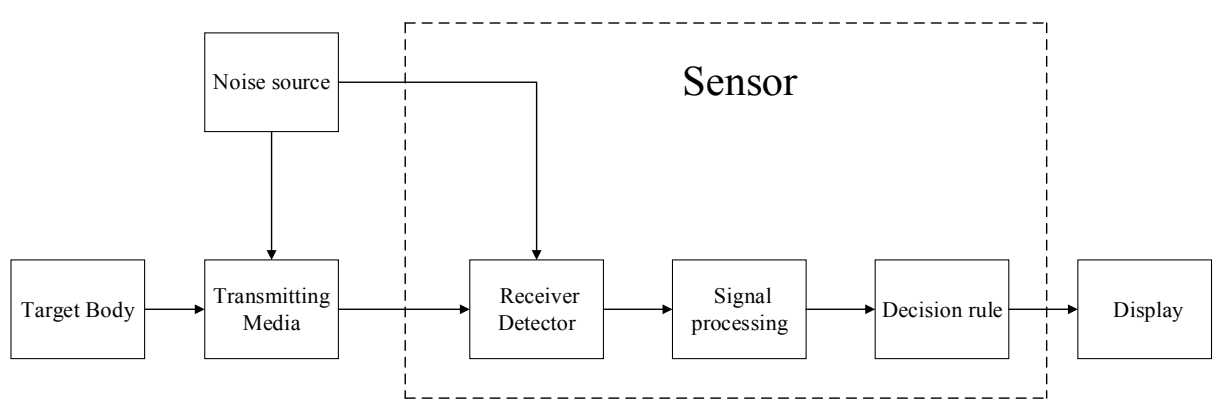

Fig. 1. A schematic diagram of sensor model with decision making function

When detecting the characteristic parameters of a random obstacle on a road, sensors will introduce continuous spreading noise in the process of sensor data transmission because of different environments. The observer, on the output end of the sensor, obtains the target entity data of narrow observation and the noise data introduced in the process of transmission. In this way, the subsequent signal processing and the decision can be considered to do detection of binary hypothesis test, estimation of determining the classification of model parameter, and multiple hypothesis testing classification and so on. The mathematical model of the sensor is shown in Figure 2.

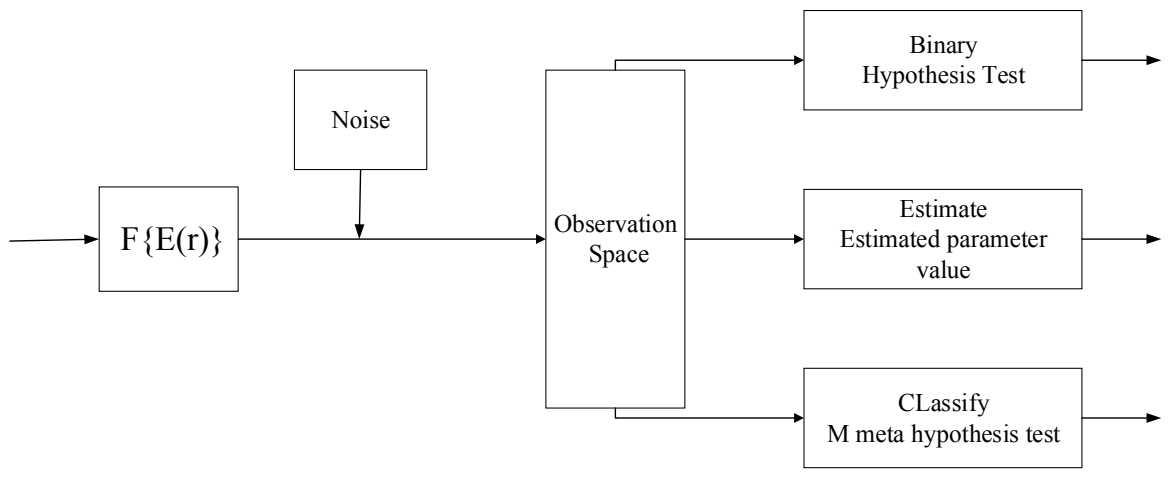

Fig. 2. The mathematical model of the sensor

Multi sensor data fusion is to integrate the scattered information measured by several different types sensors used for various purposes or those used for the same purpose but installed at different angles. And then, the advanced data processing technol- 
ogy is applied for comprehensive processing of the measured data. Thus, we can remove the redundant data and contradictory data jointly detected by several sensors in the unit of time. By combining the complementary variation reduction of the whole system, we optimize the performance indexes of the system, and enable the system more fully to obtain sensing data, so as to obtain the consistent interpretation and description of the measured entity. Multi sensor information fusion will be fused on different levels of information, such as data layer, feature layer, and decision layer.

The data fusion based on multi sensor technology is actually that the data detected by sensors are classified according to the general characteristics of the object to be measured. The redundant data and contradictory data jointly detected by several sensors in the unit of time are removed. We reserve and integrate the useful data that can highlight the measured object, and get the significant features of the measured object, easy to identify and judge. The data fusion process is shown in Figure 3.

Data fusion will have corresponding fusion relation in data layer, feature layer and decision level. Data layer is shown between data and data pairs, feature layer is displayed between feature and feature pair, and decision level is represented between decision and decision pair. The fusion relationship from the input to the output layers is shown in Figure 4.

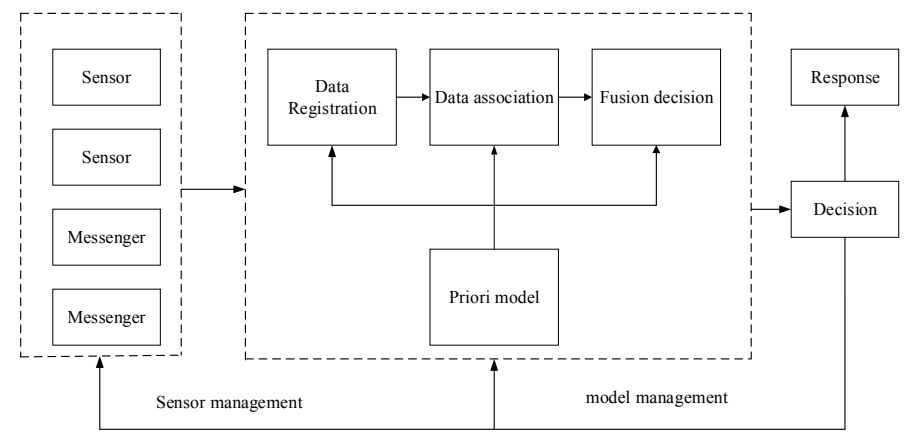

Fig. 3. Data fusion and processing process

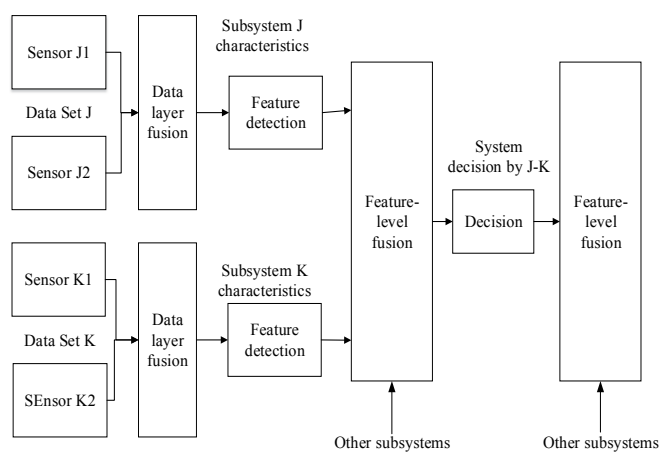

Fig. 4. The characteristic curve of the three parameter Logic static project 
From the perspective of unmanned vehicle navigation and obstacle avoidance, if there are different data fusion systems, it will also have different positioning and navigation results. The multi sensor data fusion system includes centralized, no feedback distributed, feedback distributed, and feedback parallel and so on multiple fusion structures. No matter which data fusion method, it begins with the data information detected by sensors. The structure of four different types of sensor data fusion systems $\mathrm{F}$ are shown in figures $5,6,7$, and 8 , respectively.

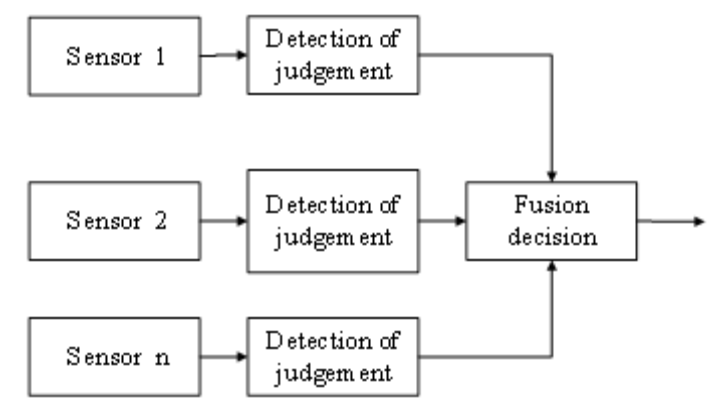

Fig. 5. Concentration type

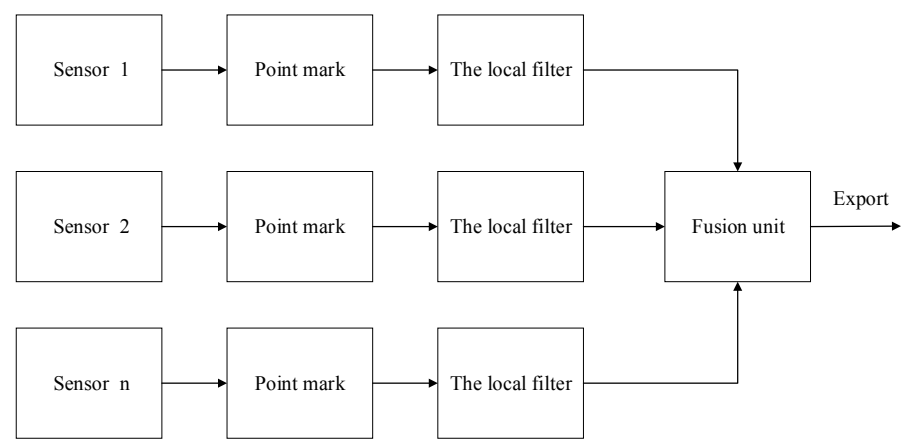

Fig. 6. No feedback distributed type

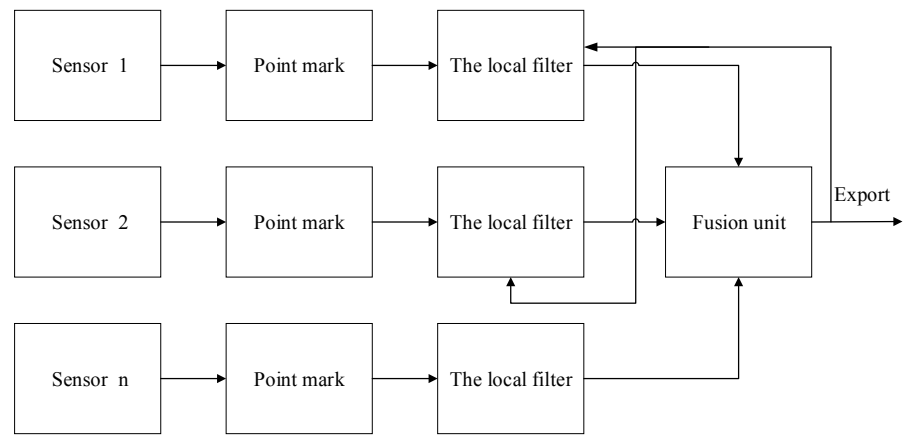

Fig. 7. Feedback distributed type 


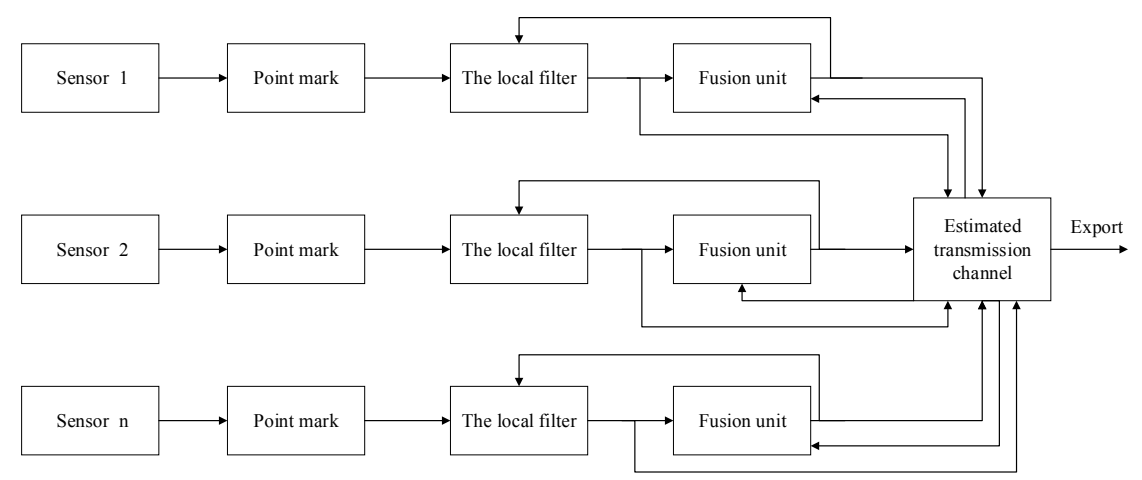

Fig. 8. Feedback parallel type

Data conversion, data inter-correlation, situation database, fusion reasoning algorithm and fusion loss are the key problems of multi sensor data fusion. The common data fusion algorithms based on multi sensor can be divided into two major categories. Kalman filtering and its evolution algorithm, Dempster Shafer evidence reasoning method, weighted average method, Bias estimation method and production rule are all stochastic methods. The theory based on fuzzy logic, artificial neural network and intelligent expert system are all artificial intelligence methods.

\subsection{Obstacle avoidance navigation algorithm of unmanned vehicle}

In this paper, the virtual force field method is used to calculate the local steering angle of an unmanned vehicle when the obstacle is detected. The sensor first of all needs to scan the environment and create a map of the scene within the range of measurements. 12 sensors are installed in the body of the unmanned vehicle, 6 in front, 2 on the left and right sides in the rear. The measurements of these ultrasonic sensors range from 5 centimeters to 4 meters.

Firstly, according to the information obtained from the 6 sensors installed in front of the unmanned vehicle, the virtual force field method is applied to calculate the repulsion force of the front of the unmanned vehicle in the middle point $\mathrm{cp} 1$ of the front wheel connection. The final repulsion is the sum of the repulsive force of 6 front sensors, and the result is expressed in (1):

$$
\overrightarrow{\mathrm{F}}_{F}=\sum_{\mathrm{i}} \frac{F_{c r} C_{i}}{d_{i}^{2}}\left[\frac{x_{i}-x_{c}}{d_{i}} \vec{i}+\frac{y_{i}-y_{c}}{d_{i}} \vec{j}\right]
$$

Secondly, in order to avoid the collision between the side car and obstacles of the unmanned vehicle, the VFF algorithm is applied to the four sensors on both sides of the unmanned vehicle. The calculation formula is as follows:

$$
\vec{F}_{s}=\frac{F_{c r} C_{i}}{d_{i}^{2}}\left[\frac{x_{i}-x_{s}}{d_{i}} \vec{i}+\frac{y_{i}-y_{s}}{d_{i}} \vec{j}\right]
$$




\section{Obstacle avoidance navigation algorithm based on multi- sensor data fusion}

\subsection{Obstacle avoidance of unmanned vehicle based on fuzzy neural network}

The data fusion model based on fuzzy neural network is shown in Figure 9.

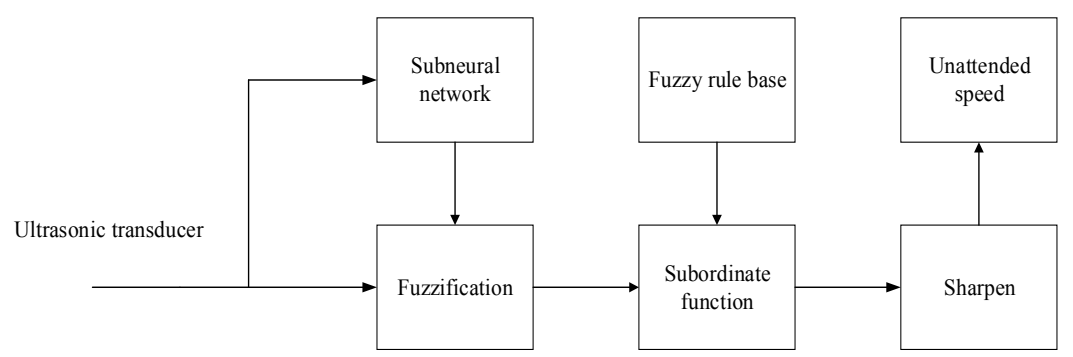

Fig. 9. The data fusion model based on fuzzy neural network

The kinematic formula of the unmanned vehicle is as follows:

$$
\mathrm{r}=r_{d}-\left(\frac{\omega_{1}}{\omega_{2}}-1\right) R_{w}
$$

In the above formula, $r_{d}$ indicates the distance between the left and right wheels and the central axis. $R_{w}$ is the radius of the wheel, $\omega_{1}$ represents the angular velocity in the moving direction of the unmanned vehicle, $\omega_{2}$ suggests the angular velocity when the unmanned vehicle turns, and $\mathrm{r}$ is the radius the obstacle avoidance circle.

\subsection{Obstacle avoidance navigation method and simulation experiment of A*VFF unmanned vehicle}

This topic uses the information with these two directions separated. When the unmanned vehicle is far away from the obstacle, the global path planning algorithm is used to navigate the direction forward, and the $\mathrm{A}^{*}$ algorithm is used in this topic. At any location in the environment, the unmanned vehicle uses global information to navigate, and the direction is to move towards the target point. When the unmanned vehicle moves closer to the obstacle during the movement, the information of the global navigation is still valid and still acts on the unmanned vehicle. When an unmanned vehicle is near an obstacle in the front or both sides, the grid around the obstacle actually has no global navigation information. In this case, the local obstacle avoidance algorithm VFF calculates a steering angle of the local position, and gives the direction adjustment information of the unmanned vehicle, so that the unmanned vehicle can safely avoid the obstacles. In addition, if the new obstacle information is detected or different from the previously detected obstacle information, the global path information will be updated. 
The project proposes the combination of the global algorithm and the virtual force field method, so as to achieve the best effect. The new theory is first to use the A* algorithm for planning of the driving path of unmanned vehicle in the global scope. As a result, a more reasonable path can be found between the starting points to the end point. In the driving process of unmanned vehicles, if we use the data of sensor to judge vehicles are getting closer to the obstacle continuously, we can bypass by using the local obstacle avoidance navigation algorithm - virtual force field. The advantages of the virtual force field method that taking into account the vehicle's own size, local obstacle avoidance and navigation accuracy is very high so that it can avoid collisions. But generally speaking, the disadvantage of virtual force field is that there will be a local minimum. For instance, when the car is driven on the obstacle areas, it will appear to be surrounded by obstacles so that it cannot normally run. At that time, we need the $A^{*} V F F$ algorithm. The principle is to let the unmanned vehicle go back to the original position by following the path entering the surrounding area. Then, it runs in accordance with the global optimization path. The new algorithm has an obvious and rapid operation rate, and it is an excellent algorithm suitable for unmanned vehicle obstacle avoidance navigation. The details are shown in Table 1 as below.

Table 1. Algorithm rate comparison e

\begin{tabular}{|c|c|c|c|c|c|}
\hline \multicolumn{7}{|c|}{ Algorithm Speed (ms) } \\
\hline & First & Second & Third & Fourth & Fifth \\
\hline Dijkstra & 5.12 & 6.98 & 7.87 & 7.32 & 6.27 \\
\hline A* & 0.56 & 1.02 & 1.32 & 0.96 & 0.85 \\
\hline A*VFF & 1.38 & 1.63 & 2.01 & 1.01 & 1.21 \\
\hline
\end{tabular}

\section{$5 \quad$ Simulation experiment and analysis}

In this paper, the multi sensor data fusion technology and obstacle avoidance navigation algorithms are studied. To verify the stability and practicability of the unmanned vehicle data fusion and navigation and obstacle avoidance algorithm, through the design of simulation environment, simulation experiment is conducted on the unmanned vehicle navigation and obstacle avoidance, and the corresponding analysis is made.

In terms of the road environment faced by unmanned vehicles, we designed the unmanned vehicle navigation and obstacle avoidance simulation environment. The unmanned vehicle sensor detects obstacles in the simulation environment, and then accurately positions the location of unmanned vehicle and obstacles around through the data fusion technology. And then, through the obstacle avoidance navigation algorithm, we plan the path for unmanned vehicle and achieve obstacle avoidance navigation purpose. Through the experiment, the obstacle avoidance navigation performance of the unmanned vehicle in the unknown environment is verified.

The simulation platform for obstacle avoidance and navigation of unmanned vehicles is composed of simulation environment settings, sensor data acquisition, multi sensor data fusion, and obstacle avoidance navigation control and data storage. Un- 
manned vehicle navigation and obstacle avoidance simulation experiment is mainly divided into two parts, hardware platform and simulation computing platform and the hardware platform is composed of simulation unmanned vehicle and road environment; the simulation platform consists of simulation environment, sensor data acquisition, multi sensor data fusion, obstacle avoidance and navigation control components, as shown in figure 10 .

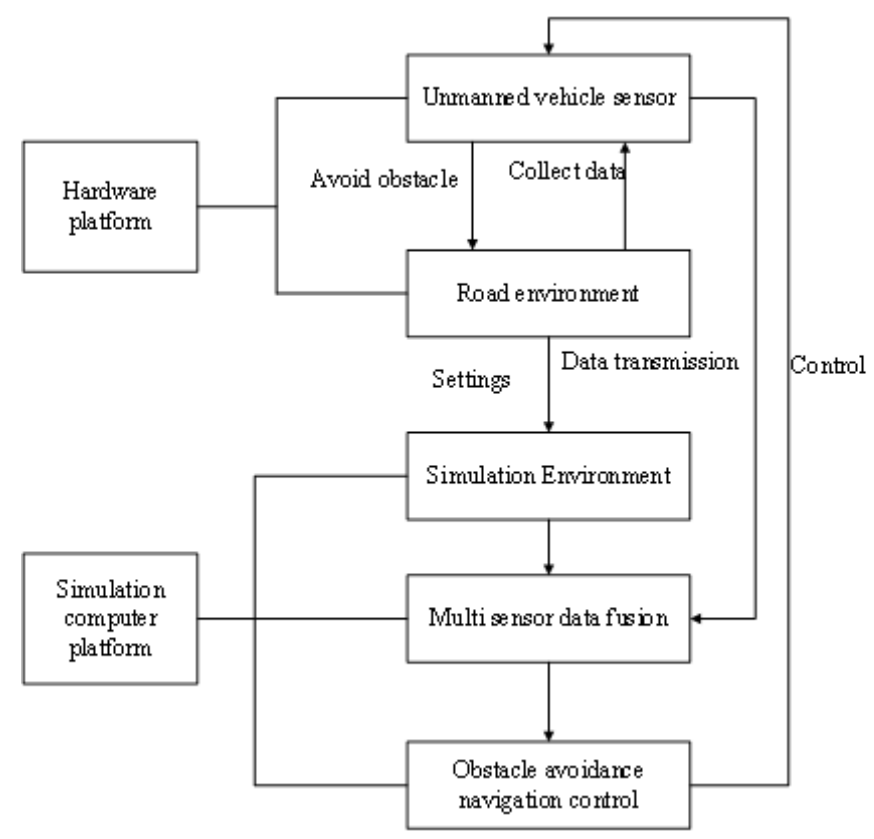

Fig. 10. Simulation experiment architecture

In the simulation experiment, the unmanned vehicle sensor is responsible for data collection, and then through the wireless transmission, data are transmitted to the simulation platform. The computing platform combines the simulation environment with the received sensor data, and then plans the obstacle avoidance path through the BP neural network data fusion procedure and obstacle avoidance navigation algorithm (A*VFF unmanned vehicle obstacle avoidance and navigation method based on fuzzy neural network). The control commands are sent to the unmanned vehicle, to command for avoidance of obstacles, and to ensure driving safety. The road environment involved in the test is divided into non-trap obstacle section, simple $U$ type obstacle section, anti U obstacle section and emergency environment section.

To test the performance of unmanned vehicle ultrasonic sensor, we conduct ranging experiments on single ultrasonic sensor, and obtain that the measurement range of single ultrasonic sensor in general is between $0.5 \mathrm{~m}$ to $5 \mathrm{~m}$. The measurement error in the distance is small, able to meet the basic requirements of the system. The measurement performance curve of the actual measurement range of the unmanned vehicle infrared sensor in the actual road conditions is shown in figure 11. 
From the performance curve shown in Figure 8, we can see that the voltage value measured by the infrared sensor does not change monotonously with the distance from the infrared sensor to the obstacles. To use monotonic performance curve, in practical applications, the measuring distance of the obstacle in $15 \mathrm{~cm} \sim 65 \mathrm{~cm}$ has good accuracy. In this paper, for BP neural network data fusion technology based on multi sensor, experiments are carried out. The training times of neural network are set to 100 thousand times and 10 thousand times, respectively, and the fusion results are shown in figures 12 and 13.

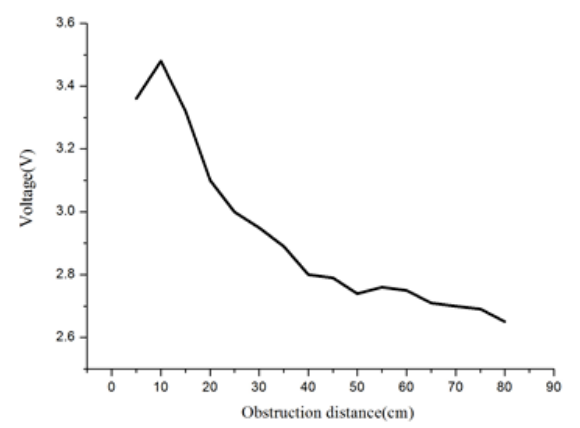

Fig. 11. Performance curve of the external sensor

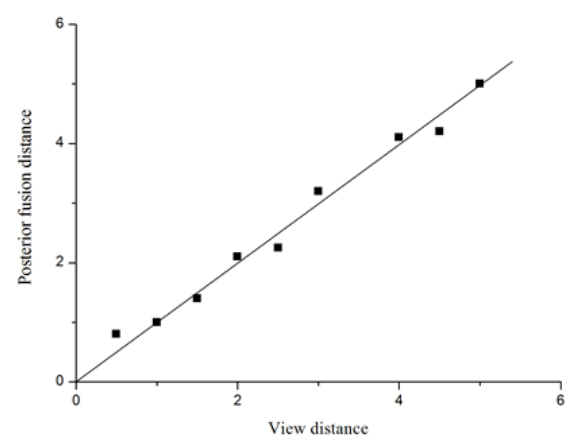

Fig. 12. 100 thousand times data fusion results of BP neural network training

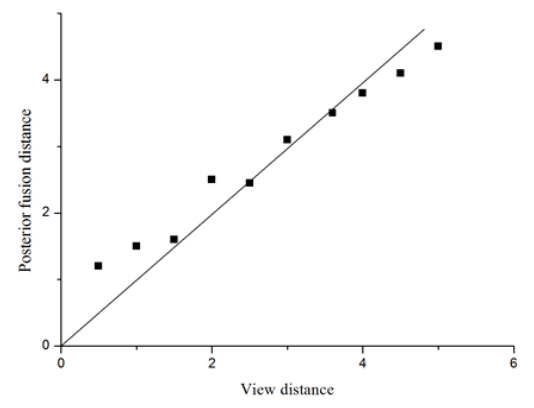

Fig. 13. 10 thousand times data fusion results of BP neural network training 
From the above chart, we can see that the data acquired by multi sensor and BP neural network data fusion algorithm are closer to the actual data. In particular, when the number of neural network training times is set to 100 thousand times, the distance after the fusion is basically consistent with the actual distance. When the number of training times is set to 10 thousand times, the running time of the program is short and the error is very small, so it can reflect the environment of the unmanned vehicle more accurately. By comparing and analyzing the results, it is shown that the navigation method of $A^{*}$ VFF unmanned vehicle based on fuzzy neural network is the best.

Generally speaking, the navigation success rate of human eyes is very low. The success rate of binocular camera and laser radar navigation can be increased by about $50 \%$. The navigation success rate of multi sensor navigation can reach over $80 \%$. Through experiment, we know that the navigation success rate of Dijkstra algorithm is about $66 \%$ and the navigation success rate of $A^{*} V F F$ algorithm based on fuzzy neural network can be further improved by about $30 \%$. Through simulation experiments, we can clearly draw the conclusion that the data fusion algorithm based on multi sensor (A*VFF algorithm based on fuzzy neural network) can significantly improve the navigation performance of unmanned vehicles. This algorithm can be used in unmanned vehicle obstacle avoidance navigation, which can decrease traffic accidents, reduce economic losses and improve transportation efficiency.

\section{Conclusion}

The multi sensor data fusion technology and unmanned vehicle obstacle avoidance navigation algorithm were studied profoundly. According to the requirements of the application of unmanned vehicle navigation and obstacle avoidance system, multi sensor data fusion technology was applied to unmanned vehicle navigation and obstacle avoidance control system. the purpose is to solve the navigation and obstacle avoidance of the unmanned vehicle. Therefore, the following conclusions are summarized:

First, with the development of science and technology and the continuous improvement of sensor performance, the application of multi sensor data fusion technology in various fields is the inevitable trend of development. The unmanned vehicle obstacle avoidance and navigation technology is the subject of great research value, as well as the key point and difficult point of research in the field of artificial intelligence control.

Second, this paper starts from the actual significance of multi sensor data fusion technology, according to the requirements of the application of unmanned vehicle navigation and obstacle avoidance system, multi sensor data fusion technology is applied to unmanned vehicle navigation and obstacle avoidance control system.

Finally, the A*VFF obstacle avoidance navigation algorithm based on fuzzy neural network is adopted. The algorithm can provide an optimal path for unmanned vehicle, it is suitable for driving in complex environment and the autonomous navigation and obstacle avoidance function of unmanned vehicle can be realized. 


\section{$7 \quad$ References}

[1] YOH-HANPAO, Phillips, S., \& Sobajic, D. Neural-net computing and the intelligent control of systems. International Journal of Control, 2016, vol. 56(2), pp. 263-289.

[2] Martín, D., García, F., Musleh, B., Olmeda, D., Peláez, G., \& Marín, P., et al. Ivvi 2.0: an intelligent vehicle based on computational perception. Expert Systems with Applications, 2014, vol. 41(17), pp. 7927-7944. https://doi.org/10.1016/j.eswa.2014.07.002

[3] Zhang, L., \& Liu, L. Research of food safety detection based on multi-sensor data fusion technology. Advance Journal of Food Science \& Technology, 2015, vol. 9(11), pp. 860865. https://doi.org/10.19026/ajfst.9.1643

[4] Kim, S., Yang, D., Kim, S., \& Jung, Y. Width estimation of stationary objects using radar image for autonomous driving of unmanned ground vehicles, 2015, vol. 18(6), pp. 711720.

[5] Wang Y.F. Design and implementation of intelligent traffic monitoring system on Thmr V platform. Computer measurement and control, 2017, vol. 25(7), pp. 106-109.

[6] Liu, W., Li, S., \& Huang, X. Extraction of lane markings using orientation and vanishing point constraints in structured road scenes. International Journal of Computer Mathematics, 2014, vol. 91(11), pp. 2359-2373. https://doi.org/10.1080/00207160.2013.813020

[7] Veum, K. S., Sudduth, K. A., Kremer, R. J., \& Kitchen, N. R. Sensor data fusion for soil health assessment. Geoderma, 2017, vol. 305, pp. 53-61. https://doi.org/10.1016/j.geo derma.2017.05.031

[8] Lin, H., \& Sun, S. Distributed fusion estimator for multi-sensor asynchronous sampling systems with missing measurements. Iet Signal Processing, 2016, vol. 10(7), pp. 724-731. https://doi.org/10.1049/iet-spr.2016.0017

[9] Li, X., Chi, J., \& Zhang, W. Target localization and tracking of unmanned mining equipment based on multi-sensor information fusion technology, 2016, vol. 6(4), pp. 147-156.

[10] Frikha, A., \& Moalla, H. Analytic hierarchy process for multi-sensor data fusion based on belief function theory. European Journal of Operational Research, 2015, vol. 241(1), pp. 133-147. https://doi.org/10.1016/j.ejor.2014.08.024

\section{Author}

Meifang Han is with Weifang University of Science and Technology, Shandong, China.

Article submitted 06 January 2018. Resubmitted 18 February 2018. Final acceptance 24 February 2018. Final version published as submitted by the author. 